type of organization in which a steady state has apparently been reached. In such an aggregation, the function $C$ must be very nearly constant over extraordinarily long intervals of time. If our galaxy is such a star cluster, it is oblate rather than globular. Assuming that it is composed of $1.5 \times 10^{9}$ stars such as our sun with an equatorial radius of 2000 parsecs $(6,600$ light years) and a polar radius of 600 parsecs, and that the mean velocity of the stars is 25 kilometers per second, the period of oscillation (if any exists) is $25,600,000$ years and the period of a circular orbit about its equator is $74,300,000$ years. If we assume a stellar density only one-fifth as great and a stellar velocity of 40 kilometers per second instead of 25 kilometers per second, the period of oscillation is $97,000,000$ years, and the period of the circular orbit about the equator is $166,000,000$ years. It should be added, however, that even though the function $C$ is a constant (or nearly constant) for the galaxy, it is not necessary that thene should be any oscillation. The moment of inertia may be a constant.

The University of Chicago.

\title{
SUBSTITUTIONS COMMUTATIVE WITH EVERY SUBSTITUTION OF AN INTRANSITIVE GROUP*
}

\author{
BY G. A. MILLER
}

It is well known how to find all the substitutions which are separately commutative with every substitution of a transitive group $G$ of degree $n$ and which involve no letter except such as are found in $G$. If $G_{1}$ is composed of all the substitutions of $G$ which omit a given letter of $G$, and if the degree of $G_{1}$ is $n-\alpha$, then all the substitutions on the letters of $G$ which are separately commutative with every substitution of $G$ constitute a subgroup $K$ of order $\alpha$, and all the substitutions of $K$ besides the identity are regular and of degree $n$.

When $G$ is intransitive, the results are not quite so elegant, but as they are often useful it seems desirable to state them

\footnotetext{
${ }^{*}$ Presented to the Society, Dec. 28, 1921.
} 
explicitly. It is evident that the direct product of the groups composed separately of the substitutions on the letters of the various transitive constituents of $G$ which are commutative with all the substitutions of these constituents is a group $K_{0}$ composed of substitutions which are separately commutative with every substitution of $G$. A necessary and sufficient condition that $K_{0}$ include all the substitutions on the letters of $G$ which are commutative with every substitution of $G$, is that $G$ have no two transitive constituents which are simply isomorphic with each other and in which the subgroups composed of all the substitutions which omit a letter correspond to such sub-groups. This results directly from the following theorem:

Theorem. A necessary and sufficient condition that there exist a substitution which interchanges $h$ systems of intransitivity and is commutative with every substitution of the intransitive group involving these systems, is that there be a simple isomorphism between the $h$ transitive groups which constitute these systems, such that a subgroup composed of all the substitutions which omit a letter of one of these $h$ transitive groups corresponds to such a subgroup of the other.

This theorem results almost directly from the fact that every automorphism of a transitive group in which a subgroup composed of all the substitutions which omit a given letter corresponds to such a subgroup, can be obtained by transforming the transitive group by substitutions involving only the letters of this group. In fact, it is evident that we can establish an automorphism of a transitive group in such a way that a substitution $t$ on the letters of the two transitive constituents thus obtained interchanges these constituents and is commutative with every substitution of the intransitive group formed by them. By transforming one of these constituents by a substitution on its own letters which transforms it into itself the isomorphism mentioned in the theorem is obtained, and $t$ is transformed thereby into a substitution which is commutative with every substitution of the intransitive group obtained in this way.

If $G$ involves $h$ transitive constituents which can be trans- 
formed transitively by means of substitutions which are commutative with every substitution of the group formed by these constituents, then these constituents can be transformed into each other according to the symmetric group of degree $h$. The total number of such substitutions on the letters of the $h$ constituents can therefore be obtained by extending the part of the direct product $K_{0}$ which relates to these constituents by means of the substitutions of this symmetric group of degree $h$. As a very special case of this theorem we have that all the substitutions which are commutative with every substitution of the intransitive group obtained by establishing a simple isomorphism between $n$ symmetric groups of degree $n>2$ and $n \neq 6$ constitute a group which is a conjugate of this intransitive group.*

From the preceding developments it results that all the substitutions which are commutative with every substitution of an intransitive group can be found by first constructing $K_{0}$ and then extending this $K_{0}$ successively by means of the symmetric groups on $h$ transitive constituents if any exist, where $h$ is always taken as large as possible. If one such $h$ is not equal to the number of transitive constituents involved in $G$, then the group composed of all the substitutions which are commutative with every substitution of $G$ is a direct product of the groups relating to the constituents which are transitively connected. While the order of each of the constituents of $G$ which can be transitively connected must be the same, those which can not be thus connected may evidently have different orders.

The University of Iluinois

* Miller, Blichfeldt, Dickson, Finite Groups, 1916, p. 41. It may be noted that the condition $n \neq 6$ should have been added there since the symmetric group of degree 6 admits outer isomorphisms. 